\title{
Regular Trends of Acousto-Optic Interaction in Terahertz Region of Electromagnetic Radiation
}

\author{
P. Nikitin ${ }^{a}, *$, V. Voloshinov ${ }^{a}$, V. Gerasimov ${ }^{b}$ And B. KNyazeV ${ }^{c}$ \\ ${ }^{a}$ Department of Physics, Lomonosov Moscow State University, 119991 Moscow, Russia \\ ${ }^{b}$ Budker Institute of Nuclear Physics, Siberian Branch, Russian Academy of Sciences, 630090 Novosibirsk, Russia \\ ${ }^{c}$ Novosibirsk National Research State University, 630090 Novosibirsk, Russia
}

\begin{abstract}
Basic problems of acousto-optic interaction in terahertz region of electromagnetic spectrum are considered. We obtained experimental results that are fitted by generalized theoretical model developed with the goals to describe the interaction in detail. Our analysis showed that crystalline germanium is one of the best materials to observe the acousto-optic interaction in the terahertz range. The carried out study proved that a germanium based acousto-optic device could be used for fast and reliable deflection of monochromatic radiation in the terahertz spectral region.
\end{abstract}

DOI: 10.12693 /APhysPolA.127.49

PACS: $78.20 . \mathrm{hb}, 42.25 . \mathrm{Fx}$

\section{Introduction}

Acousto-optic (AO) interaction is widely used to control such parameters of electromagnetic radiation as direction of propagation, phase, frequency, intensity and state of polarization [1-3]. Since nature of the phenomenon does not depend on frequency of the electromagnetic radiation, AO devices have already been used in the ultraviolet, visible, infrared and even in the terahertz $(\mathrm{THz})$ spectral ranges. Usually, these devices are AO modulators, deflectors and tunable filters. However, so far there are no publications describing these devices and their operation in the $\mathrm{THz}$ range. Unfortunately, a number of papers related to the AO interaction in the $\mathrm{THz}$ frequency range of the electromagnetic radiation is limited to a few contributions $[1,2]$. The main reason for this drawback is a number of disappointing features of the $\mathrm{AO}$ interaction in the $\mathrm{THz}$ region. These features namely are the low diffraction efficiency and the substantial divergence of light, i.e. the $\mathrm{THz}$ radiation, due to its relatively long wavelengths $\lambda[3-5]$. The second characteristic feature is related to the strong absorption of light at the values of the absorption coefficient $\alpha=4 \pi n^{\prime \prime} / \lambda$ of the order from 1.0 to $10 \mathrm{~cm}^{-1}$. Here $n^{\prime \prime}$ is the imaginary part of the refractive index $n=n^{\prime}-\mathrm{i} n^{\prime \prime}$, which is usually much smaller than the real part of it $n^{\prime \prime} \ll n^{\prime}$. The purpose of our study was generalization of the well-known theory of the AO interaction over media in part transparent in the $\mathrm{THz}$ region. Experimental examination of the AO interaction in solid and liquid media promising for the $\mathrm{AO}$ applications in the $\mathrm{THz}$ region was also one of the goals of the research.

\section{Theory}

We follow general approach of the plane-wave theory of the AO interaction [3] to formulate the total electric

*corresponding author; e-mail: nikitin.pavel.a@gmail.com field $E$ in terms of complex amplitudes of the diffracted $C_{1}$ and transmitted $C_{0}$ light waves:

$$
\boldsymbol{E}(x, y)=\sum_{p=0,1} C_{p}(x) \exp \left(\mathrm{i}\left(\boldsymbol{k}_{p} \boldsymbol{r}-\omega_{p} t\right)\right),
$$

where $\boldsymbol{k}_{p}=k\left\{\cos \theta_{p}, \sin \theta_{p}, 0\right\}$ is the wave vector and $\omega$ is the angular frequency of light and $x$ - the coordinate across the sound beam. In our analysis, changes in the wave number $k$ are neglected. Note that Eq. (1) has the essential limitation in the direction of propagation $\theta_{p} \ll 1$, which is satisfied only at the quasi-orthogonal and the quasi-collinear geometry of AO interaction.

It is known that a plane acoustic wave changes permittivity $\varepsilon$ of a medium:

$$
\varepsilon=n^{\prime 2}-2 \mathrm{i} n^{\prime} n^{\prime \prime}+2 n^{\prime} \Delta n^{\prime} \cos (\boldsymbol{K} \boldsymbol{r}-\Omega t),
$$

where $\boldsymbol{K}$ is the acoustic wave vector which is equal to $K\{1,0,0\}$ at the quasi-collinear geometry, and $K\{0,1,0\}$ at the quasi-orthogonal geometry.

In order to derive a system of coupled wave equations, one has to substitute Eq. (2) in the Maxwell equations and collect terms with equal time dependent exponential factors. This procedure is possible only if the angular frequencies satisfy the condition $\omega_{n}=\omega_{0}+n \Omega$. The Bragg matching condition is evaluated by means of the mismatch parameter $\Delta k=k_{1}-k_{0}-K$ dependent only on $x$-component [3]. Therefore, we obtain the following equations describing the AO interaction in the Bragg regime:

$$
\left\{\begin{array}{l}
\partial C_{0} / \partial x=0.5\left[-\alpha_{0} C_{0}+\mathrm{i} q_{0} C_{1} \exp (\mathrm{i} \Delta k x)\right], \\
\partial C_{1} / \partial x=0.5\left[-\alpha_{1} C_{0}+\mathrm{i} q_{1} C_{1} \exp (-\mathrm{i} \Delta k x)\right],
\end{array}\right.
$$

where $\alpha_{p}=2 k_{p} n^{\prime \prime} / n^{\prime} \cos \theta_{p}$ is the absorption coefficient and $q_{p}=k_{p} \Delta n^{\prime} / n^{\prime} \cos \theta_{p}$ is the well-known coupling coefficient.

Analytical solution of the system Eq. (3) with the boundary conditions $C_{0}(0)=1$ and $C_{1}(0)=0$ is as follows:

$$
C_{0}(L)=\left[\cos \left(\frac{s L}{2}\right)-\frac{2 \mathrm{i} \Delta k+\alpha_{0}-\alpha_{1}}{s} \sin \left(\frac{s L}{2}\right)\right]
$$




$$
\begin{gathered}
\times \exp \left(\frac{\left(2 \mathrm{i} \Delta k-\alpha_{0}-\alpha_{1}\right) L}{4}\right), \\
C_{1}(L)=\mathrm{i} \frac{q_{1}}{s} \sin \left(\frac{s L}{2}\right) \\
\quad \times \exp \left(-\frac{\left(2 \mathrm{i} \Delta k+\alpha_{0}+\alpha_{1}\right) L}{4}\right),
\end{gathered}
$$

where $s=\sqrt{q_{1} q_{1}+\Delta k^{2}+\left(\alpha_{1}-\alpha_{0}\right)\left(4 \mathrm{i} \Delta k+\alpha_{0}-\alpha_{1}\right) / 4}$ and $L$ is a width of the sound beam usually equal to a length of a piezoelectric transducer.

Evaluation of the obtained expressions (4) and (5) is rather complicated. However, if the angles $\theta_{0}$ and $\theta_{1}$ are narrow and the acoustic angular frequency is much smaller than that of light, then $\alpha_{p}=\alpha=2 k_{0} n^{\prime \prime} / n^{\prime}$ and $q_{p}=q=k_{0} \Delta n^{\prime} / n^{\prime}$. Consequently, the derived expressions (4) and (5) become simpler:

$$
\begin{aligned}
& C_{0}(L)=\left[\cos \left(\frac{s L}{2}\right)-\mathrm{i} \frac{\Delta k}{s} \sin \left(\frac{s L}{2}\right)\right] \\
& \quad \times \exp \left(\frac{(\mathrm{i} \Delta k-\alpha) L}{2}\right), \\
& C_{1}(L)=\mathrm{i} \frac{q}{s} \sin \left(\frac{s L}{2}\right) \exp \left(\frac{(-\mathrm{i} \Delta k-\alpha) L}{2}\right),
\end{aligned}
$$

where $s$ is defined as $s=\sqrt{q^{2}+\Delta k^{2}}$.

It is obvious that the absorption of light results in a decrease of the optimal length of the AO interaction $L^{\mathrm{opt}}$ required reaching maximum of the diffraction efficiency. Here we consider the AO interaction between a plane electromagnetic wave incident on ultrasound at the Bragg incidence $(\Delta k=0)$ and a sound beam having constant acoustic power. In case of the orthogonal AO interaction geometry we have $q=2 A / \sqrt{L}$, where $A=$ const. In case of the collinear geometry $q$ remains constant $q=2 B$, where $B=A / \sqrt{l}$ and $l$ is a size of the acoustic beam in the direction orthogonal to the plane of the AO interaction. We found that the intensity of the diffracted light $I_{1}=\left|C_{1}\right|^{2}$ in the quasi-orthogonal and the quasi-collinear interaction is given by the following equation in terms of the dimensionless parameters $X_{1}=\alpha / A^{2}, Y_{1}=A^{2} L_{1}$, $X_{2}=\alpha / B$ and $Y_{2}=B L_{2}$ :

$$
I_{1}^{\text {ort }}=\mathrm{e}^{-X_{1} Y_{1}} \sin ^{2} \sqrt{Y_{1}}, \quad I_{1}^{\text {col }}=\mathrm{e}^{-X_{2} Y_{2}} \sin ^{2} Y_{2} .
$$

Now we fix the dimensionless absorption coefficient $X$ to find an optimal value $Y^{\text {opt }}$ of the dimensionless interaction length $Y$. We proved that an analytical solution $Y^{\text {opt }}(X)$ can be found only in the case of low diffraction efficiency, when $Y \ll 1$, namely $Y_{1}^{\text {opt }}=1 / X_{1}$ and $Y_{2}^{\text {opt }}=2 / X_{2}$. It is clear that numerical approach is applicable for arbitrary values of the parameter $X$. The results of numerical calculations are plotted in Fig. 1 in $\log -\log$ scale.

We managed to reveal correlation between the optimal length $L^{\mathrm{opt}}$ and the absorption coefficient $\alpha$ in the case of a low diffraction efficiency $L_{1}^{\text {opt }}=1 / \alpha$ and $L_{2}^{\text {opt }}=2 / \alpha$. The relation between $L^{\text {opt }}$ and $\alpha$ provides estimation of the optimal size of the crystal even if the coupling coefficient is unknown. That is why

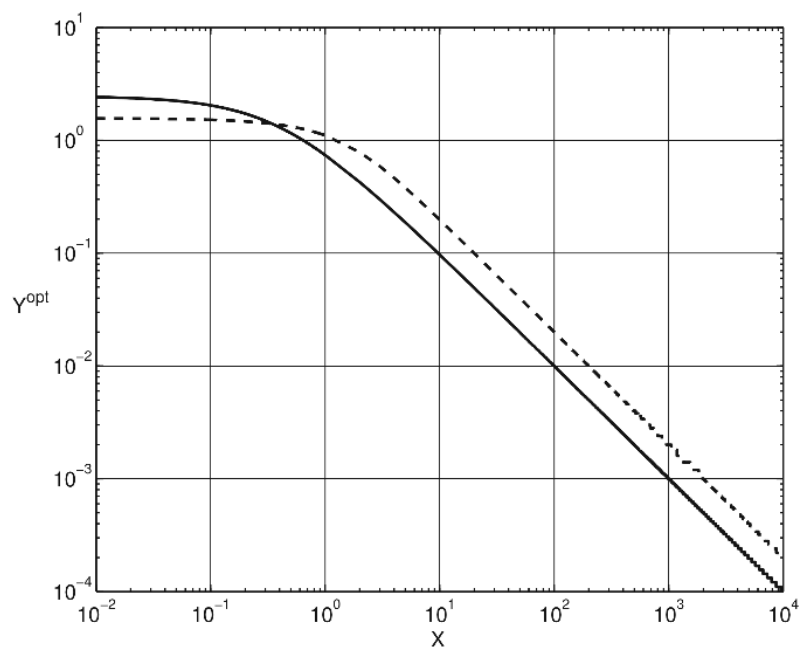

Fig. 1. Dependence of the optimal dimensionless length of AO interaction on the dimensionless absorption coefficient. The solid line corresponds to the orthogonal geometry, while the dashed line describes the collinear geometry.

the intensities of diffracted light are given by $I_{1}^{\text {ort }}=$ $A^{2} / \alpha e$ and $I_{1}^{\text {col }}=4(B / \alpha e)^{2}=I_{1}^{\text {ort }} 4 / \alpha l e$. We used these expressions to evaluate the efficiency of the AO interaction in the terahertz region of spectrum.

\section{Experimental results}

We measured transparency of a few AO materials in the $\mathrm{THz}$ region of spectrum in a preliminary experiment carried out by means of the Novosibirsk free-electron laser (FEL) [4]. It was found that the majority of known AO-materials were opaque. Therefore, basic experiments were carried out in AO cell fabricated of a high-resistive single crystal germanium [5].

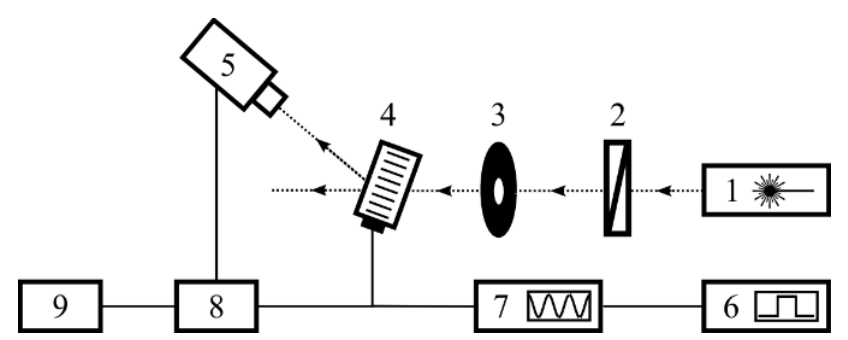

Fig. 2. Scheme of the experimental setup: (1) $\mathrm{THz}$ free-electron laser, (2) polarizer, (3) diaphragm, (4) AO deflector, (5) Golay detector, (6) pulse generator, (7) high-frequency generator, (8) lock-in amplifier, and (9) personal computer.

The scheme of the experimental setup is shown in Fig. 2. Polarization and aperture of the THz FEL radiation (1) was set by the polarizer (2) and the diaphragm (3). The radiation passed through the AO cell (4) and it was registered by the Golay detector GC1T (5). Electrical signal at the RF-input of the AO cell was formed by the pulse generator (6) and the highfrequency generator (7). The lock-in amplifier (8) was 
applied to extract and amplify a diffracted signal coming from the Golay detector. The experimental data was collected and processed using the personal computer (9).

The germanium crystal was chosen for the analysis due to its high refractive index $n=4$ and relatively low absorption coefficient $\alpha=0.751 / \mathrm{cm}$. Unfortunately, in our experiment, the length of the piezoelectric transducer $L=2.0 \mathrm{~cm}$ was longer than the optimal length $L^{\mathrm{opt}}=1.3 \mathrm{~cm}$. We measured two different dependences in the carried out experiments [5]. The goal of the first experiment was related to measurement of the frequency dependence of the Bragg angle $\theta(F)$ in the cell. We registered the dependence in the acoustic frequency band 25-38 MHz. The second experiment gave evaluation of the acoustic frequency bandwidth of diffraction $\Delta F$ registered at a fixed angle $\theta$ of the radiation incidence. It was proved that the examined device could be used as a reliable deflector providing scanning of the monochromatic $\mathrm{THz}$ radiation at the wavelength $\lambda=140 \mu \mathrm{m}$. The diffraction efficiency $\xi=I_{1}(L) / I_{0}(L)$ was equal to $(5.0 \pm 0.3) \times 10^{-4}$ at $1.0 \mathrm{~W}$ of the driving electrical power. Spatial resolution of the deflection was evaluated as $N=\Delta F \tau=8$ pixels, where the quick-action of the device was about $\tau=2 \mu$ s and $3 \mathrm{~dB}$ acoustic frequency bandwidth was $\Delta F=4 \mathrm{MHz}$ at the central frequency of about $30 \mathrm{MHz}$. It should be noted that the registered value of the diffraction efficiency correlated with the general theoretical estimation. As for a more detailed information on the experimental investigation as well as on a graphical representation of the obtained results, they may be found in Ref. [5].

In the most recent experiments, we examined the $\mathrm{AO}$ interaction in a number of non-polar liquids such as cyclohexane $\mathrm{C}_{6} \mathrm{H}_{12}$, white spirit WS and tetra-chloro-methane $\mathrm{CCl}_{4}$. The study proved the general trend that the efficiency of the AO diffraction in germanium was one order higher than that in the liquids: $\xi=(2.7 \pm 0.2) \times 10^{-5}$ for $\mathrm{C}_{6} \mathrm{H}_{12}$ and $\xi=(5.0 \pm 1.2) \times 10^{-5}$ for WS. Moreover, the acousto-optic deflection of light in $\mathrm{CCl}_{4}$ was not detected at all. We note that the large values of experimental errors originated from intensity fluctuations of the $\mathrm{THz}$ radiation at the operating frequencies of about 10-15 Hz. We had to use these extremely low operating frequencies because they corresponded to maximal sensitivity of the used Golay detector GC-1T [6].

\section{Conclusion}

To summarize the foregoing, we state that the $\mathrm{AO}$ interaction in the $\mathrm{THz}$ range of electromagnetic spectrum is rather weak and difficult to observe due to the strong absorption and divergence of radiation. More sophisticated theoretical and experimental investigations should be performed to understand principal aspects of the diffraction phenomenon in the $\mathrm{THz}$ region. It is expected that further investigations will result in development of new and efficient methods to control parameters of the $\mathrm{THz}$ radiation. Another direction of future investigations is related to search of new acousto-optic materials having high diffraction efficiency in the $\mathrm{THz}$ range. As for the crystalline germanium, for today it seems that it is the best available material to realize the acousto-optic interaction in the terahertz spectral range.

\section{Acknowledgments}

The research was supported by the grant N 14-1200380 of the Russian Scientific Fund.

\section{References}

[1] T. Vogel, G. Dodel, Infrared Phys. 25, 315 (1985).

[2] W. Durr, W. Schmidt, Int. J. Infrared Millim. Waves 6, 1043 (1985).

[3] V.I. Balakshy, V.N. Parygin, L.E. Chirkov, Physical Principles of Acousto-Optics, Radio i Svyaz, Moscow 1982.

[4] B.A. Knyazev, G.N. Kulipanov, N.A. Vinokurov, Meas. Sci. Technol. 21, 054017 (2010).

[5] V.B. Voloshinov, P.A. Nikitin, V.V. Gerasimov, B.A. Knyazev, Yu.Yu. Choporova, Quant. Electron. 43, 1139 (2013).

[6] www.tydexoptics.com. 